\title{
6 Seele, Geist und kosmische Intelligenz - Strukturen des Bewusstseins
}

\author{
Joachim Galuska
}

Unsere Bewusstheit kann ein Schlüssel sein, uns aus den alltäglichen Identifizierungen unseres Ich-Bewusstseins herauszulösen und eine innere Verankerung in einer tieferen und weiteren Bewusstseinsstruktur einzunehmen.

Lassen Sie sich einmal versuchen zu spüren, dass Sie gerade anwesend sind, vergegenwärtigen Sie, dass Sie gerade da sind, anwesend sind, sonst nichts, einfach bewusst anwesend sein.

Für mich ist dies Präsenz, das bewusste Spüren unseres lebendigen Anwesendseins. Dieses unmittelbare Empfinden von Anwesendsein weist auf etwas Wesenhaftes hin. Unser Wesen wird durch Anwesendsein spürbar: „An“ kann gefühlt werden wie „Da“: Dasein, gegenwärtig sein. „Wesend“ kann gefühlt werden wie: "geschehend, seiend, inne seiend des eigenen Wesens".

\section{Seele}

Anwesend fühlt und spürt unsere Seele sich selbst, vergegenwärtigt sie sich ihre ursprüngliche Eigenart. Unsere Seele ist fühlend, spürend, rezeptiv und wesend, geschehend, im Fluß. Es ist eine Art fließendes Spüren des Erlebens. Wenn wir diese innere Haltung weiter erforschen, finden wir eine Reihe von Eigenschaften, die wir vertiefen können und dann in einer zunehmenden Reinheit erleben können:

Reine Bewusstheit, reine Achtsamkeit, innere Klarheit, Weite und Raumhaftigkeit dieses Bewusstseins, dass wie ein offener, klarer, unendlich weiter Bewusstseinsraum empfunden wird, innerhalb dessen alle Erlebnisweisen erscheinen. Dieses Bewusstsein ist also offen und leer, frei von unseren Identifizierungen und allen einzelnen Erlebnisinhalten. „Leere Weite“ wird eine seiner Eigenschaften genannt. Und 
dieser Bewusstseinsraum ruht in sich selbst, er trägt in sich selbst Frieden und Stille. Angesichts der Offenheit des Erlebens wird eine tiefe Verbundenheit empfunden mit den Inhalten des Erlebens, insbesondere mit denen, die sich auf solche Wesensmerkmale beziehen. Wir können diese auch als essenzielle Qualitäten, als „transpersonale“ Qualitäten oder als Seelen-Qualitäten bezeichnen. So lernen wir zu unterscheiden zwischen unserer Wesenstiefe und unserer Oberfläche, unseren Grundwerten und unseren oberflächlicheren Interessen. Hier können wir spüren, was geschieht, wenn wir unsere Souveränität verlieren und uns in einer Teilidentität, einer Rolle oder gar einem Konflikt zwischen Teilrollen verlieren und wie sich dies wieder auflösen kann. Ein wesentliches Merkmal dieser Bewusstseinshaltung besteht darin, dass wir den Prozess der Identifizierung erkennen, der Identifizierung in unserem Denken und Fühlen, mit unseren Konzepten von uns selbst, unseren Konzepten von der Welt, unseren Überzeugungen und Anschauungen. Und wir können uns desidentifizieren und dennoch einen Halt in uns finden. Diese Bewusstseinshaltung nenne ich gerne unsere Seele, unser Wesen, unser essenzielles Selbst (Galuska 2003) (s. Abb. 9).

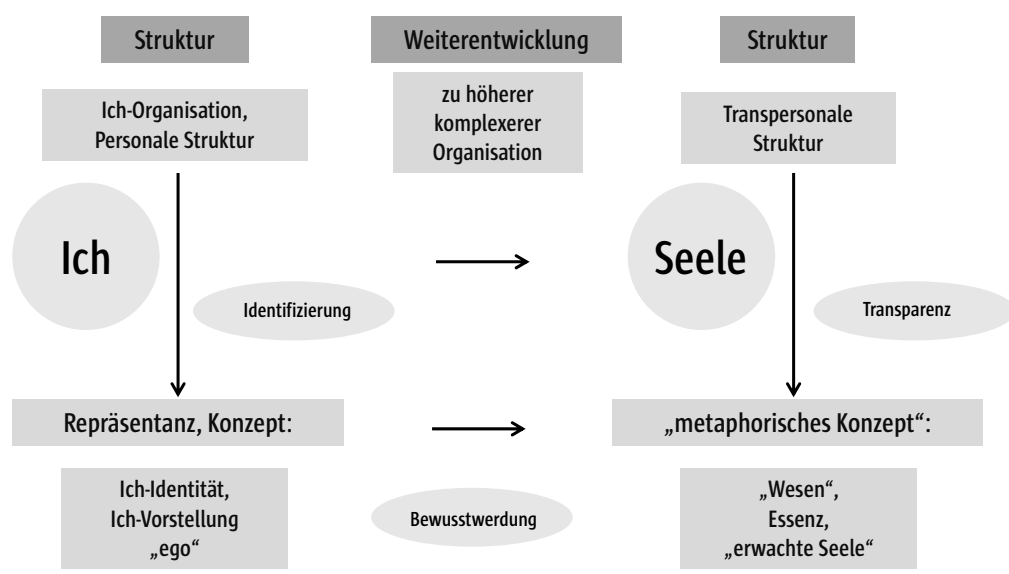

Abb. 9 Ich und Seele

Ich meine damit einen Seelenbegriff, der nicht auf die Funktionen der Psyche reduziert wird. In der modernen Psychotherapie ist der Seelenbegriff inzwischen weitgehend abgelöst worden von den Begriffen „Selbst“, „Ich“, „Identität“ und „Persönlichkeit“, die die zusammenfassenden und steuernden Funktionen der Psyche bezeichnen. Unsere Seele ist aber mehr als der Ort unserer inneren Konflikte zwischen unseren verschiedenen Persönlichkeitsinstanzen, zwischen Bewusstem und Unbewusstem. Sie ist mehr als der Ort unserer inneren Reflexion über unsere Einstellungen, Motivationen und Kognitionen. Wir haben in unserer abendländischen Tradition eine Geschichte des Seelenbegriffes, die sehr viel weiter geht. Die Seele hatte immer eine religiös-spirituelle Dimension. Sie war so etwas wie ein Mittler zwischen Diesseits und Jenseits, ein Bote zwischen den Welten, wie es sich im Bild des Seelenvogels, der zwischen Himmel und Erde fliegt, ausdrückt. „Seele“, stammt als Wort vom urgermanischen „Saiwolo“ $\mathrm{ab}$, die vom See her kommende, dem Aufenthaltsort der Seele vor der Geburt und nach dem Tod. Sie ist dem Menschen als Lebensatem eingehaucht, was sich im griechischen Wort „Psyche“, Atem, Lebenskraft zeigt. So heißt es in der Schöpfungsgeschichte: 
„Und er, Gott, bildete den Menschen, Staub vom Acker, er blies in seine Nasenlöcher Hauch des Lebens und der Mensch wurde zum lebenden Wesen“ (2. Buch Moses, Genesis 2, 7, Übersetzung von Martin Buber).

Wir Menschen haben die Seele also immer religiös oder spirituell interpretiert, und zwar nicht nur in den Religionen, sondern auch in der Philosophie, von Platon bis zu Augustinus, die die Seele als etwas Ewiges und Göttliches betrachteten. Erst die moderne Philosophie hat die Seele verweltlicht, bis hin zur Neurophilosophie, die Seele und Geist lediglich als Absonderungen des menschlichen Gehirns versteht. Aber für mich besitzt die Seele eine Offenheit für die Transzendenz, denn eine ihrer wichtigsten Eigenschaften ist vielleicht ihre Transparenz, die gleichzeitige Offenheit für die Erscheinungsformen der Welt und für unser Aufgehobensein im Seinsgrund, im Göttlichen, Absoluten und Unbekannten. Dort ist keine Struktur, keine Form, keine Bewegung, kein Konzept, sondern etwas, was all dies transzendiert, ein Schweben, ein Geschehen. Die Erscheinung der Welt ist dagegen das Bewusstsein einer spezifischen Wirklichkeit, einer konkreten Form: ein individuelles Leben, eine persönliche Wirklichkeit, eine konkrete Situation.

Wir könnten sagen, dass sich das Unbekannte und Absolute in das individuelle und persönliche Leben und Erleben wandelt und als solches erscheint. Leere wandelt sich also in Form und Form wandelt sich wieder in Leere, um einmal die große Metapher des Buddhismus zu verwenden. Die Struktur dieses Wandlungsprozesses von Leere in Form und wieder von Form in Leere ist für mich eine der Grundstrukturen unserer Seele. Die Seele ist für mich die individuelle Art und Weise, wie das Unbekannte und Absolute sich manifestiert und allem Erlebten seine Gestalt gibt. Die Seele erkennt also das Unbekannte, Formlose oder Göttliche und die Welt der Formen, Erscheinungen und Daseinsweisen als zwei Seiten ihres Wesens. Ich habe diese Bewusstseinsstruktur der Seele und ihre Anwendungen für die Psychotherapie, die Wirtschaft und die persönliche Bewusstseinsentwicklung in den letzten Jahren ausführlich beschrieben und möchte dies an dieser Stelle nicht weiter ausführen (Galuska 2003a, 2003b, 2004, 2005, 2010).

\section{Geist}

Unsere Bewusstseinsentwicklung besitzt jedoch eine Tendenz zum Überschreiten der gegenwärtigen Grenzen unseres Bewusstseins, eine Tendenz zur Transzendenz, könnte man sagen, und zur Suche nach dem uns noch Unvertrauten und Unbekannten. Das Seelenbewusstsein wendet sich also dem Absoluten und Unbekannten selbst zu. Die spirituellen Wege haben Methoden entwickelt, das Unbekannte direkt und unmittelbar zu realisieren und dann diese Position einzunehmen, in die Welt zu schauen als das nicht näher Konzeptionalisierte, als das Offene, als das Geheimnisvolle, das alles durchdringt. Das Unbekannte dieser Art enthält damit nicht nur das primitive Unbewusste unserer persönlichen oder artspezifischen Vergangenheit, sondern auch unser Potenzial, unsere Zukunft und das Unbeschreibliche und Mysteriöse unserer Gegenwart, die wir realisieren können als Ausdruck des Wirkens einer unermesslichen Tiefe des evolutionären Geschehens. Um einen solchen weiteren Schritt in der Bewusstseinsentwicklung zu erreichen, löst sich das Seelenbewusstsein von allem Bekannten, von allen Formen und Inhalten des Erlebens, von allem, was sichtbar, fühlbar oder denkbar ist, lässt sich fallen, schweben. Es folgt der Auflösung aller Formen dahin, wo nicht etwas ist, wo nichts ist, völlig gelöst und frei (s. Abb. 10). 
Das Bewusstsein des Unbekannten ist wie ein gelöstes, freies Schweben in der Formlosigkeit. Die Sprache unseres Seelenbewusstseins beschreibt dies zunächst negativ als das Un-bekannte, Un-begründete, Un-getrennte, Form-lose, Eigenschafts-lose, Zeit-lose, Raum-lose, A-kausale, Nichts-hafte. Eine andere Beschreibung stellt das Urhafte in den Vordergrund: das Ursprüngliche, das Urtümliche, den Urgrund.

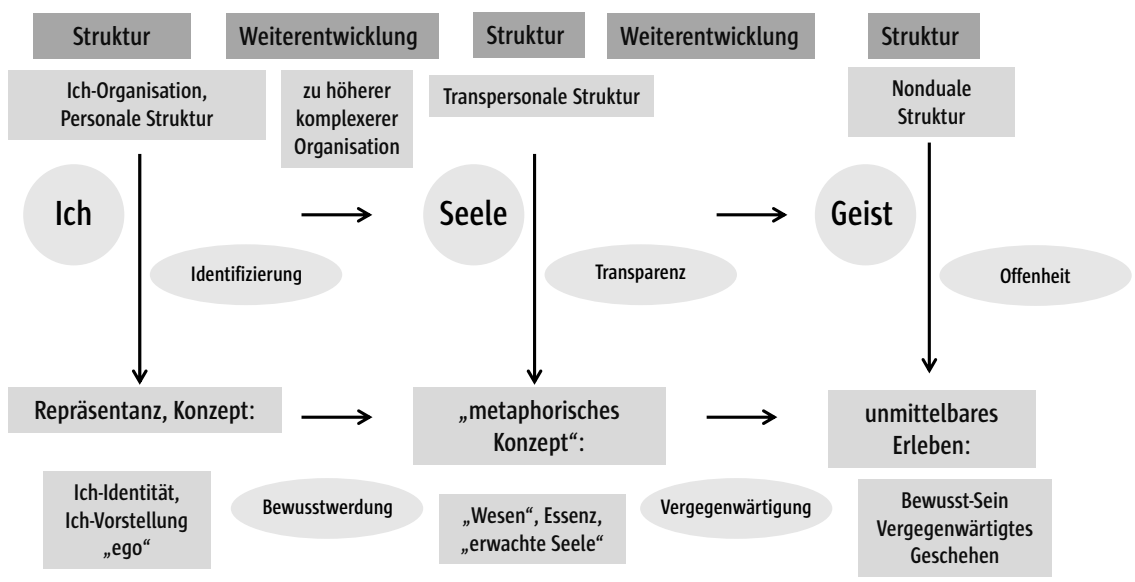

Abb. 10 Ich, Seele und Geist

Hier gibt es keine Trennung mehr, kein Bewusstsein, das das Unbekannte beobachtet, sondern das Bewusstsein realisiert sich selbst als ursprünglich, formlos, frei und gelöst. Es vergegenwärtigt sich seiner selbst als „So“, als Ausdruck des Unbekannten, das alles unmittelbar durchdringt, ohne Hindernis, wie gesagt wird. Hier ist Leere Form, Form ist Leere. Form wird realisiert als unmittelbarer Ausdruck der Leere. Jeder Moment wird vergegenwärtigt als der, der er ist, in seiner So-heit, als „so“, unmittelbar, vollständig. Dieses Bewusstsein ist nondual, weil keine Trennung mehr da ist, kein Gegenüber. Alles geschieht aus sich selbst heraus, ohne sichtbare Ursache, a-kausal.

\section{David Loy (1988) differenziert drei Aspekte der Nondualität:}

- Nonduale Wahrnehmung bedeutet, keine Trennung von Beobachter und Beobachtetem zu erleben, von Wahrnehmendem und Wahrgenommenen. Das Wahrgenommene und Erlebte realisiert sich selbst, vergegenwärtigt sich selbst als So.

- Nonduales Denken bedeutet, kein Bewusstsein eines Denkers von Gedanken zu haben, sondern die Vergegenwärtigung des spontanen Flusses des Denkens zu sein, strömende geistige Kreativität, die sich aus sich selbst heraus entfaltet.

- Nonduales Handeln bedeutet Nichttun im Tun, keinen Täter zu empfinden, der Taten vollbringt, keine Absicht, die Ziele verfolgt, sondern vergegenwärtigtes, spontanes fließendes Geschehen zu sein, anstrengungslos, aus sich selbst heraus steuernd.

Zu ergänzen wäre noch die Nondualität von Dualität und Nondualität. Damit ist gemeint, dass auch das dualisierende Erleben der Welt, das konzeptionalisierende Erleben, das vergegenständlichende Erfassen der Wirklichkeit erfahren und erkannt 
wird als das, was es ist, eben duales Erleben, das damit dual vergegenwärtigt wird. Ich-Bewusstsein wird vergegenwärtigt als Ich-Bewusstsein, Seelen-Bewusstsein als Seelen-Bewusstsein, Gut und Böse als mentale Kategorien, Worte als Worte und all dies als Ausdruck des Unbekannten, als Manifestation des Göttlichen als die Welt, wenn man so will.

Ich nenne die Struktur dieser inneren Bewusstseinsverankerung gerne „Geist“. Während die Seele eher wie ein offenes Gefäß für jede Erfahrung ist, die sie in ihrem inneren Raum verortet, einordnet, fühlt und bewertet, vergegenwärtigt der nonduale Geist sich jeden Moment unmittelbar, ohne jede Beschränkung, so wie er ist, als der, der er ist. Er schmeckt ihn sozusagen direkt. Während die Seele sich innerlich berühren lassen kann und sich anrühren lassen kann, in Resonanz treten kann und andere beseelte Wesen berühren kann, braucht dies der nonduale Geist nicht, weil er unmittelbar vergegenwärtigt, sozusagen die Spiegelung des anderen im eigenen Geist erkennt, ohne Bewertungen, Vorlieben und Abneigungen, ohne innere Verpflichtungen, wie sie die Seele noch fühlt.

\section{Kosmische Intelligenz}

Unser Geist realisiert diese gesamte Entwicklung des Erwachens zu uns selbst, die Struktur dieser Entwicklung. Und er erkennt das Offene der Evolution, das sich selbst Kreierende, das nicht Vorherbestimmte und damit auch das Offene unserer Bewusstseinsentwicklung. Und er erkennt dies als Gefüge einer umfassenderen Intelligenz, die wir vielleicht als „,kosmische Intelligenz“ bezeichnen könnten, die diesen gesamten Bewusstseinsprozess konfiguriert. Der nonduale Geist kann erkennen, dass der gesamte Kosmos, das Unergründliche, Unbekannte und auch sein eigenes Bewusstsein Teil einer Intelligenz ist, die weit über die nonduale Struktur unseres Bewusstseins hinausreicht und eben auch sie konfiguriert. Geist und Kosmos entfalten sich gemäß einer eigenen Intelligenz, die weit über das hinausgeht, was unser Bewusstsein erfassen kann (s. Abb. 11). Es ist die Intelligenz, gemäß derer sich der Kosmos, die Evolution und unser Bewusstsein ereignet, gemäß derer also alles so geschieht, wie es geschieht: Diese Intelligenz ist nicht das Unbekannte, das Absolute, das Göttliche, das Nonduale, sondern sie konfiguriert das Bewusstsein so, dass sie durch die-

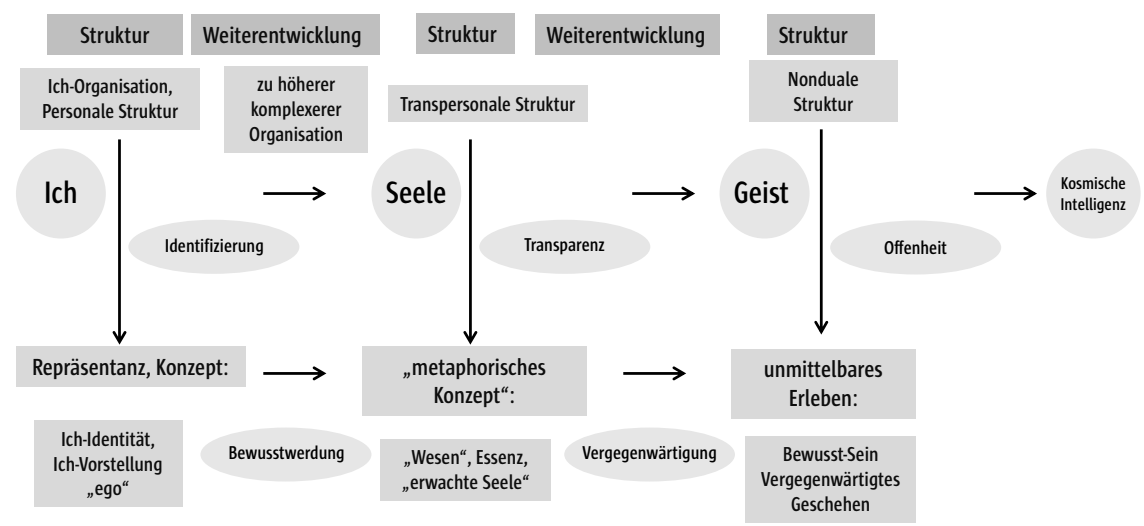

Abb. 11 Ich, Seele, Geist und kosmische Intelligenz 
se Tore erahnt oder erschlossen werden kann. In meinem Verständnis schwebt also der sich entfaltende nonduale Geist in dieser umfassenderen Intelligenz, dieser MetaOrdnung, die selbst das nonduale Bewusstsein eben erst nondual sein lässt. Ich verwende hier die Worte „Geist“ und „Intelligenz“, um eine Differenzierung herzustellen und damit verschiedene Ebenen unseres Bewusstseins beschreiben zu können.

\section{Bewusstseinswissenschaften}

Aus der Sicht der Neurobiologie wären Ich, Seele, Geist, Intelligenz oder Bewusstsein überhaupt nur Modelle eines Gehirns von sich selbst. Sie wären nur ausgedrückte Strukturen eines neuronalen Systems, das ein Bild von sich selbst erzeugt. Aber anders herum betrachtet ist das Gehirn, so wie wir es verstehen, selbst lediglich ein konzeptionalisiertes Phänomen. Wir untersuchen eigentlich nur ein Modell eines Gehirns, wenn wir ein Gehirn untersuchen, so wie wir eben Konzepte von der Wirklichkeit bilden und diese dann überprüfen. Hirnforscher untersuchen also nicht als Gehirne andere Gehirne und deren Bewusstsein, sondern sie untersuchen mit Hilfe einer bestimmten Bewusstseinsstruktur, in der Regel eines konzeptualisierenden Ich-Bewusstseins, die Außenseite eines lebendigen, fühlenden, bewussten Nervengewebes. Wir projizieren unsere Denk- und Bewusstseinsstruktur in unser Gehirnverständnis, wie wir sie in jedes unserer Verständnisse hineinprojizieren. Das heißt, wir können unser Gehirn und die Ergebnisse der Hirnforschung nur mit Hilfe und auf die jeweilige Art unserer Bewusstseinsstruktur erfassen. Dann aber glauben wir, wie es eben bei einer Projektion üblich ist, das Gehirn wäre so und produziere eben unser Bewusstsein wie Ich, Wille, Emotionen, Erinnerungen usw. auf eine biologische Weise. Nein - wir können es genau anders herum sehen: Wir interpretieren die biologischen Prozesse und auch unsere Bewusstseinsprozesse auf die jeweilige Art und Weise unserer Denk- und Bewusstseinsstruktur. Ich, Seele und Geist sind also nicht Produktionen unseres Gehirns, sondern die Ursache für die Interpretation der neurobiologischen Prozesse und Ergebnisse. Ich-Bewusstsein, Seelen-Bewusstsein und nondualer Geist gehen mit einem jeweils anderen subtileren oder komplexeren Gehirnverständnis einher. Deswegen sagt Wilfried Belschner zu Recht, dass das Bewusstsein des Forschers eine unabhängige Variable darstellt. Sein Bewusstseinszustand hat Auswirkungen auf seine Forschungsergebnisse und vor allem auf die Interpretationen seiner Ergebnisse. Ken Wilbers Quadrantenmodell kann uns zeigen, dass der Forscher im Zustand des integralen Bewusstseins, also eines weit entwickelten Ich-Bewusstseins zunächst entscheidet, durch welchen Blickwinkel er schaut: aus der Perspektive des Gehirns auf Gehirn und Bewusstsein, oder aus der Perspektive des Bewusstseins auf Bewusstsein und Gehirn, ähnlich wie bei Welle und Teilchen. Wir brauchen also in den Wissenschaften und insbesondere in den Bewusstseinswissenschaften eine ständige Reflexion und ein Gespräch darüber, aus welcher inneren Bewusstseinsverankerung wir gerade sprechen. Wir brauchen somit eine Relativierung der Betrachtung in Bezug auf unsere Blickwinkel und die Struktur des Bewusstseins, das diese Blickwinkel einnimmt. Jede Wissenschaft selbst ist ja nur eine Bewusstseinsaktivität, könnte man sagen, und im Fall der Bewusstseinswissenschaften richtet sie sich auf sich selbst und seine eigene Entwicklung und ist immer abhängig von dem jeweiligen Bewusstseinszustand und der jeweiligen Bewusstseinsentwicklung. Möglicherweise spüren wir im Zustand des Seelen-Bewusstseins auf unserem Grund das unbekannte und intelligente Geschehen unserer neuronalen Prozesse, die einer eigenen 
Intelligenz folgen, selbst organisiert und aus sich selbst heraus sich bewegend. Die Intelligenz, in der das Bewusstsein und das Geschehen der neuronalen Prozesse aufgehoben ist und denen sie folgen, ist eins, denn sie kann nur mit Hilfe oder über unser Bewusstsein erfasst werden. Sie konfiguriert Gehirn und Geist als Gehirn und Geist, als sich selbst realisierendes, erwachendes Nervensystem, das seine eigene Grundlage erforscht und sich aneignet und damit zum Mitschöpfer der weiteren Bewusstseinsentwicklung wird.

„Aufzuwachen“ in unserem Sinne ist eine Eigentümlichkeit und Eigengesetzlichkeit dieser Intelligenz. Von außen her betrachtet, entfaltet ein Lebewesen so seinen Innenraum, einen „Weltinnenraum“ (Rilke), eine innere Vorstellungswelt von der Wirklichkeit, die ihm Orientierung gibt und mehr Spielraum, sich darin zu bewegen und zu handeln. Und auch die Fähigkeit des erwachenden Bewusstseins sich zu steuern, sein Leben zu gestalten, sich weiterzuentwickeln und selbst seine eigene Entwicklung frei, kreativ oder gar kunstvoll zu beeinflussen ist intelligent, ist Ausdruck dieser Intelligenz.

Das, wo sich alle Bewusstseinsforscher heute treffen könnten, seien sie Psychologen, Sozialwissenschaftler, Neurobiologen, Ethnologen oder spirituelle Sucher, könnte das Nicht-Wissen im guten Sinne sein, der Respekt vor dem Unbekannten, der uns den gemeinsamen Crund aufzeigt und spüren lässt, jenseits aller Konzepte, Schulen und Wissenschaften. Und dieser Grund besitzt, wenn er einmal erkannt und gespürt wird, eine enorme Präsenz, die so etwas wie einen gemeinsamen Bezug darstellen kann. Krishnamurti (1980) fragte in seinen Vorträgen immer wieder, wie das Leben eigentlich ist, wenn es nicht an eine Idee verpflichtet ist, eine Religion, ein Konzept, wenn es nicht in Bildern, Vorstellungen und Illusionen lebt, die uns voneinander abgrenzen oder gegeneinander aufbringen. Dann entstehe ein „Sense of nothingness“ - ein Gespür für „not a thing“, keine Verdinglichungen, Identifizierungen, Bewertungen, Konstruktionen mehr, sondern Offenheit, Bewusstheit, innere Stille. Dies eröffne dann eine Liebe, Freiheit und Intelligenz, die nicht mehr korrumpierbar sind. Und ich verbinde damit die Hoffnung auf einen gemeinsamen freien kreativen Geist unseres Menschseins, mit dem wir eine lebendige Bewusstseinswissenschaft gestalten und entwickeln können, die uns inspiriert und hilft, unser Leben auf diesem Planeten in Größe, Fülle und dem Potenzial unserer Spezies gemäß zu gestalten.

\section{Literatur}

Galuska I (2003a) Die erwachte Seele und ihre transpersonale Struktur. Transpersonale Psychologie und Psychotherapie $2 / 2003,6-17$

Galuska I (2003b) Grundprinzipien einer transpersonal orientierten Psychotherapie. In: Galuska I (Hrsg.) Den Horizont erweitern. 38-62. Ulrich Leutner Verlag Berlin

Galuska I (2004) Spirituelles Bewusstsein in Wirtschaftsprozessen. In: Galuska I (Hrsg.) Pioniere für einen neuen Geist in Beruf und Business. 11-33. I. Kamphausen Verlag Bielefeld

Galuska I (2005) Auf dem Weg zu einer Psychotherapie des Bewusstseins. In: Galuska J, Pietzko A (Hrsg.) Psychotherapie und Bewusstsein. 17-35. I. Kamphausen Verlag Bielefeld

Galuska J. (2010) Wirtschaften mit Geist und Seele. In: Galuska I (Hrsg.) Die Kunst des Wirtschaftens. 121-133. I. Kamphausen Verlag Bielefeld

Loy D (1988) Nondualität. Krüger Verlag

Krishnamurti I (1980) Talks in Saanen. Tape 7. Is there anything sacred in life. Auditorium Netzwerk 


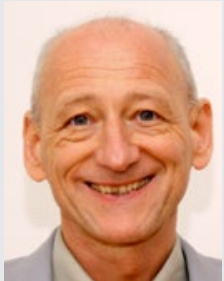

Dr. med. Joachim Galuska

Facharzt für Psychosomatische Medizin und für Psychiatrie und Psychotherapie, Geschäftsführer und Ärztlicher Direktor der Heiligenfeld Kliniken in Bad Kissingen und Waldmünchen. Herausgeber u.a. der Bücher: Die Kunst des Wirtschaftens (2010), Psychotherapie und Bewusstsein (2005), Gründer der Zeitschrift Bewusstseinswissenschaften und der Stiftung Bewusstseinswissenschaften. 